Canadian Oncology

Nursing Journal

Revue canadienne

de soins infirmiers

en oncologie

Volume 27, Issue 2 • Spring 2017

elSSN: 2368-8076 


\title{
The role of oncology nurse navigators in enhancing patient empowerment within the diagnostic phase for adult patients with lung cancer
}

\author{
by Gaya Jeyathevan, Manon Lemonde, and Angela Cooper Brathwaite
}

\begin{abstract}
Objective: The objective of this study was to explore the role of oncology nurse navigators (ONN) in enhancing patient empowerment for adult patients with lung cancer during the diagnostic phase of cancer care.
\end{abstract}

Design: A qualitative descriptive design was used. Data were gathered through individual patient interviews and a focus group with the ONNs.

Results: Eight individuals participated: four adult patients with lung cancer and four oncology nurse navigators. Themes regarding the clinical functions of the ONN role in enhancing patient empowerment were identified: patient advocate, educational support, personalized symptom management, and resource navigation.

Conclusion: ONNs are in a key position to adapt their practice to the emotional and supportive care needs required to promote patient and family empowerment.

Key words: nurse navigators, patient empowerment, cancer, diagnostic phase

\section{INTRODUCTION}

$\mathrm{T}$ The diagnostic phase of cancer is a stressful period for patients. Furthermore, the healthcare system is very complex and patients must interact with many healthcare professionals within multiple health services across the cancer care continuum. According to Moseholm et al. (2016), undergoing diagnostic evaluation for cancer was associated with a

\section{ABOUT THE AUTHORS}

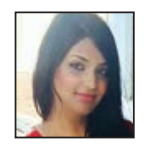

Gaya Jeyathevan, PhD(c), Institute of Health Policy, Management and Evaluation, University of Toronto, Toronto, ON M5T 3M6

Correspondance : Institut des politiques, de la gestion et de l'évaluation de la santé, Université de Toronto, Toronto, $\mathrm{ON}$

Email: gaya.jeyathevan@mail.utoronto.ca

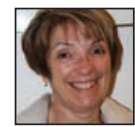

Manon Lemonde, RN, PhD, University of Ontario Institute of Technology, Oshawa, ON L1H 7K4

Email: manon.lemonde@uoit.ca

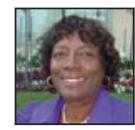

Angela Cooper Brathwaite, RN, PhD, Adjunct Professor, University of Ontario Institute of Technology, Oshawa, ON L1H 7K4

Email: angela.cooperbrathwaite@utoronto.ca

https://doi.org/10.5737/23688076272164170 high prevalence of anxiety and depression, which affected health-related quality of life. They reported the prevalence of clinical anxiety prior to knowing about the diagnosis was $32 \%$ in patients with cancer and $35 \%$ in patients who received a non-cancer diagnosis. That patients awaiting a diagnosis of cancer are at risk for increased psychosocial morbidity has also been reported by others investigators (Bultz \& Carlson, 2005; Drageset, 2012).

During the diagnosis of cancer, treatment and follow-up care, most patients can benefit from empowerment, as they continuously experience changes during the long trajectory from diagnosis to palliative care (Rosenbaum \& Spiegel, 2002). Patient empowerment is described as a process in which healthcare providers provide patients with opportunities and knowledge to develop skills and confidence to move from being a passive recipient of care to an active partner in their healthcare (World Health Organization, 2009; European Network on Patient Empowerment, n.d.). Ongoing advice and encouragement from the healthcare team are critically important for empowering patients. However, many healthcare professionals do not have time nor the expertise to train and counsel patients (McCorkle et al., 2011).

Cancer navigation has emerged as a model of care within North America to promote patient and family empowerment (Fillion et al., 2012). In Canada, professional navigation within the oncology field is usually practised by registered nurses (Pedersen \& Hack, 2010). Professional nurse navigators assist the patients and their families at many points along the cancer journey, providing a single point of contact and bridging the patients and their families to the interdisciplinary healthcare team, the cancer centre, and community services (Cook et al., 2013). Although nurse navigation is acknowledged as a fundamental element of an integrated system of cancer care, this navigation process is not yet delivered nationally in a standardized way (Cancer Care Ontario, 2010). There is a paucity of research on the effectiveness of nurse navigators' role on patients' experience (Cantril \& Haylock, 2013; Fillion et al., 2012; Pedersen \& Hack, 2011). To our knowledge, no Canadian studies have shown how oncology nurse navigators (ONNs) have promoted empowerment for patients and their families during the diagnostic phase of cancer. The purpose of the study was to explore the role of oncology nurse navigators (ONN) in enhancing patient empowerment for adult patients with lung cancer during the diagnostic phase of cancer care. 


\section{CONCEPTUAL FRAMEWORK}

Concepts of the Bi-Dimensional Framework developed by Fillion et al. (2012) underpinned this study, and guided data analysis and interpretation of the results. The Bi-Dimensional Framework was developed in early 2012 to bring clarity to the roles of professional nurse navigators within Canada, as well as assist ONNs in being more efficient and less challenged in terms of setting priorities and making decisions, while facing demands from the healthcare system and patients. The framework consists of two-theoretical dimensions: (a) facilitating continuity of care, and (b) promoting patient empowerment. This paper focuses on the second dimension: promoting patient empowerment, which is composed of the three related concepts of active coping, self-management, and supportive care.

\section{METHODS}

\section{Study Design}

This study used a qualitative descriptive design to explore the role of ONNs in enhancing patient empowerment for adult patients with lung cancer during the diagnostic phase of cancer care at Durham Regional Cancer Centre (DRCC) in Ontario. This design was chosen because it allows for the presentation of the study and results in everyday language (Sandelowski, 2000). There was also a paucity of qualitative research on the roles and impact of ONNs during the diagnostic phase of cancer. The design included a combination of sampling, data collection, analysis and representational techniques. Individual interviews with patients and a focus group with ONNs were conducted using semi-structured open-ended questions. This study received ethical approval from the Lakeridge Health and the University of Ontario Institute of Technology Research Ethics Boards. Participants provided informed consent prior to the interviews and focus group.

\section{Recruitment}

Convenience sampling was used to recruit the eight participants. Using patients' records and preliminary telephone screening, the ONNs identified potential patients based on the eligibility criteria. The eligibility criteria for patients included: adult men and women 18 years or older, referred to the diagnostic assessment program at the DRCC with a suspicion of lung cancer, had at least two contacts with the ONN prior to data collection, and fluent in English. Following an invitation letter to potential participants, interested persons who met the selection criteria were contacted by the principal investigator (PI) via telephone to inform and recruit them into the study. Eligibility criteria for ONNs included: working at the DRCC within the diagnostic assessment program, provided healthcare to patients, completed lung-specific courses, and held the designation of Certified in Oncology Nursing (Canada, CON(C)). Recruitment occurred between January 2013 and March 2013. Recruitment concluded when successive interviews became repetitive and no new responses or themes emerged (Creswell, Plano Clark, Gutmann, \& Hanson, 2003).

\section{Data Collection}

Individual patients participated in a 15- to 20-minute interview by answering open-ended questions. ONN participants engaged in a focus group, answering open-ended questions, which lasted approximately 35 minutes. The patient interviews and the ONN focus group were conducted by the PI, who had no previous connection with participants. The interview guide was developed using a detailed description of the concepts within the Bi-Dimensional Framework. The interview questions consisted of concepts such as: (1) active coping (i.e., how the ONNs provided help in learning coping mechanisms); (2) self-management (i.e., how the ONNs provided support for patients to proactively self-manage their altered health); and (3) supportive care (i.e., how the ONNs addressed patients' evolving needs). Probes or recursive questioning were used during the interviews and focus group to explore particular issues in greater depth and verify the interviewer's understanding of the information being collected (Patton, 2015). Individual interviews and the focus group were audio-recorded; recordings were transcribed verbatim. Table 1 contains a sample of the interview questions.

\section{Data Analysis}

Data collection and analysis were carried out in an iterative manner. The PI verified the accuracy and precision of the transcripts. Themes were created from participants' narratives through the iterative process of thematic analysis (Graneheim \& Lundman, 2004). A subset of interview transcripts was

Table 1: List of examples of open-ended questions from interview guide

\section{Active Coping:}

1a. Patient version: Did you receive any education and support by your nurse navigator to enhance your self-care? Please provide an example of a time when this helped to enhance your sense of personal health maintenance.

1b. ONN version: How do you actively take steps to facilitate problem solving and decision making?

\section{Self-Management:}

2a. Patient version: How has the nurse navigator assisted you in adjusting to and managing your altered health state? Did your informal caregivers (i.e. family, friends) have necessary information about your health and managing your symptoms that they needed in order to help you out?

2b. ONN version: How often do you assess or monitor the symptoms of the patients? In what ways do you provide symptom management to ameliorate the patient's overall cancer care and experience?

\section{Supportive Care:}

3a. Patient version: Did your nurse navigator refer you to services within your community to address any unmet supportive care needs?

3b. ONN version: What kind of services do you provide to patients to meet their physical, emotional, psychosocial, social, and spiritual needs? 
initially coded by the PI, giving full attention to all data. Then the PI developed a coding framework for the remaining transcripts. The other two researchers reviewed the transcripts, summarized the informational content in the data to identify themes, compared accuracy of findings and interpretation of the data, and explored and resolved any inconsistencies in coding. This ensured rigor and enhanced reflexivity. Segments of data, an idea or word conveying an idea, were identified prior to being subsumed under a theme. A theme included configuration of segments of data. The codes were finally clustered

\begin{tabular}{|c|c|}
\hline Characteristics of Patients & $n=4 ; n$, Range \\
\hline \multicolumn{2}{|l|}{ Sex } \\
\hline Male & 2 \\
\hline Female & 2 \\
\hline Age & $58-71$ \\
\hline \multicolumn{2}{|l|}{ Symptoms experienced } \\
\hline Shortness of breath & 2 \\
\hline Weight loss & 2 \\
\hline Fatigue & 2 \\
\hline Swollen joins & 2 \\
\hline No symptoms & 2 \\
\hline \multicolumn{2}{|l|}{ Education } \\
\hline High School & 3 \\
\hline Undergraduate/College & 1 \\
\hline \multicolumn{2}{|l|}{ Relationship status } \\
\hline Married & 3 \\
\hline Divorced & 1 \\
\hline \multicolumn{2}{|l|}{ Employment status } \\
\hline Retired & 3 \\
\hline Employed & 1 \\
\hline Characteristics of Oncology Nurse Navigators & $n=4 ; n$, Range \\
\hline \multicolumn{2}{|l|}{ Sex } \\
\hline Male & 0 \\
\hline Female & 4 \\
\hline Age & $39-50$ \\
\hline Years working in oncology & $4.5-20$ \\
\hline \multicolumn{2}{|l|}{ Education } \\
\hline Undergraduate/College & 3 \\
\hline Post-graduate & 1 \\
\hline \multicolumn{2}{|l|}{ Employment status } \\
\hline Part-time & 2 \\
\hline Full-time & 2 \\
\hline
\end{tabular}

into categories and key themes were identified. 'Outsider' checks of the developing analysis occurred through conversations within the research team to maximize credibility and trustworthiness (Toma, 2011). All three researchers explored various thematic maps until consensus was reached and themes were agreed upon.

\section{FINDINGS}

Eight individuals participated in this study (four adult patients with lung cancer, whose right-lobes were removed, and four oncology nurse navigators). Characteristics of the participants are reported in Table 2.

\section{Clinical Functions of Oncology Nurse Navigators}

Synthesis of the findings resulted in the identification of core clinical functions within the ONN roles which enhance patient empowerment. Table 3 presents the themes with selected participants' quotes. These core clinical functions within the ONN roles were determined based on patients' perceptions of how ONNs' roles impacted the patients' and their families' empowerment, with respect to the concepts of active coping, self-management, and supportive care. The following clinical functions of the ONN role for enhancing patient empowerment were identified: patient advocate, educational support, personalized symptom management, and resource navigation. Subsumed under active coping was patient advocate and educational support. Self-management included personalized symptom management. Supportive care consisted of resource navigation.

\section{Active coping}

An integral role of the ONN is to take proactive steps in an attempt to remove the stressors from the patients during the diagnostic phase. This is done by being a patient advocate and providing educational support, thus assisting the patients to cope with their illness.

Patient advocate.

There was consensus among patients and ONN participants that ONNs played a significant role in identifying the needs of patients and by advocating for them and their families throughout the diagnostic process. ONNs reported that they became the "voice for the patient to get them to where they needed to be", using other hospital departments or community health care services. One participant added, "being an advocate was not going to change the course of the disease but helped them cope with what they were experiencing at the time."

\section{Educational support.}

Participants across both groups noted the significant role that ONNs played by providing educational support so the patients and their families obtained the information and support needed when making vital decisions about their health care. Educational support was also described as detailed explanations of medical terms and side effects of treatments to enhance the patients' knowledge about the tests during the diagnostic phase. By providing such educational support, the patients reported they felt more at ease to actively cope with 
Table 3: Themes and representative quotes of participants

\begin{tabular}{|c|c|}
\hline Themes & Source of Representative Quotes \\
\hline $\begin{array}{l}\text { Educational } \\
\text { Support }\end{array}$ & $\begin{array}{l}\text { ONN said: } \\
\text { "The nurse always spends time with the patient on their own just to review the information the doctor's given and again go through } \\
\text { the synopsis of any investigations that they may be going through." } \\
\text { Patient said: } \\
\text { "Well it was a big cache of information. She went through some of it with me, especially the tests, but there are all kinds of } \\
\text { brochures about lung cancer." }\end{array}$ \\
\hline $\begin{array}{l}\text { Personalized } \\
\text { Symptom } \\
\text { Management }\end{array}$ & $\begin{array}{l}\text { Patients said: } \\
\text { "When I knew I have little tiny bumps in my mouth, and she explained to me I could either use baking soda or mouth wash. And } \\
\text { then she actually said we'd get you something for that. Then she had prescribed a mouth wash for me. So, any little things, there } \\
\text { was always something she'll be there for." } \\
\text { "She told us (informal caregiver-wife) how to take care of me, like from keeping from getting a cold, or just like different things } \\
\text { that they bring to your attention." }\end{array}$ \\
\hline $\begin{array}{l}\text { Resource } \\
\text { Navigation }\end{array}$ & $\begin{array}{l}\text { ONN said: } \\
\text { "A lot of people that were diagnosed with cancer, or were looking at being diagnosed with cancer, that was not always their focus. } \\
\text { It was 'what am I going to do with my kids or job?" They were not even thinking of what their treatment was going to be like. Zoning } \\
\text { in on that and seeing what their needs were at that moment may not be even cancer related. Just knowing that there was help, } \\
\text { resources and people made the process easier for them." } \\
\text { Patient said: } \\
\text { "Such a wide variety of services were available... I certainly got all my needs met. I was not lacking in anything. I phoned for a ride } \\
\text { to come here today." }\end{array}$ \\
\hline
\end{tabular}

their disease and changes they were experiencing during this process. The ONNs reported that they provided educational support from the first consult, so the patients were prepared to solve any problems that may arise and make decisions concerning their health care. The ONNs also reported that they "continued to be an ongoing resource" for the patients to lessen the impact of the stressors they were experiencing and take control of their lives.

\section{Self-Management}

Self-management proved to be a key concept in this study. In order to reinforce the patients' abilities to accept the illness and regain control, the ONNs took the initiative to provide personalized symptom management to help them cope with physical changes. Patient participants understood their health condition and its effect on their body. They participated in the decision making with healthcare providers by contributing to the care plan and indicating their preferences. They also felt more informed about choices and treatment options.
Personalized symptom management.

All patient participants reported that the ONNs effectively took measures to actively ensure that they understood specific self-efficacy enhancing techniques to cope with their cancer, such as symptom management. The patients further reported that the ONNs took initiative to explain to their family members how to monitor and manage symptoms, as well as how to appropriately provide care for them. In addition to providing symptom management techniques, both participant groups reported that the ONNs also assessed symptoms during every consult. The ONNs kept track of the patients' symptoms by asking the patients to fill out a symptom management scale, called the 'Edmonton Symptom Assessment Scale' (ESAS), and provided advice or support to patients on how to proactively manage their altered state and symptoms based on the findings from the scales. Identifying patients' needs was also significant when educating the patients on the various specific efficacy-enhancing techniques to cope with their cancer and plan their care accordingly. 


\section{Supportive care}

In order to meet the supportive care needs of the patients and families during the diagnostic phase of cancer care, the ONNs provide resource navigation accordingly.

\section{Resource navigation.}

The ONNs reported that they were proactive in identifying the supportive care needs of the patients so as to address the general distress and unmet needs, as well as reinforce empowerment. For example, the ONNs referred patients to resources / community services in order to address and meet patients' and their families' needs. All participants agreed that if the patients and/or family members needed specific services, the ONNs facilitated access to these services either within the hospital or the community outreach programs. Although the ONNs felt they facilitated resource navigation, the patients also reported that their ONNs provided supportive care by referring them to community services.

\section{DISCUSSION}

The current study explored the roles of ONNs in enhancing patient empowerment of adult patients with lung cancer during the diagnostic phase of cancer. Using a qualitative descriptive design, four clinical functions of the ONN role for enhancing patient empowerment were identified. These functions were patient advocate, educational support, personalized symptom management, and resource navigation. In combination, these functions profiled an interconnected model that placed the patients with cancer and their family members at the centre of care during the diagnostic phase of the disease continuum. This was the first qualitative study to add a broader understanding of the oncology ONN roles in enhancing patient empowerment within the diagnostic phase of cancer, from the perspectives of lung cancer patients and ONNs.

Collectively, this study demonstrated that ONNs acted like a bridge between the health system and patients. According to Wilcox and Bruce (2010), the main goal of the newly developed role of an ONN is to advocate for the patients and their families by coordinating and enhancing care among all interdisciplinary team members. With a nursing background and a comprehensive understanding of oncology components and the diagnostic assessment phase, the ONNs were highly competent in acting as an advocate for their patients. The ONNs explained that by maintaining a good relationship with the patients and their families, they were able to identify the patients' needs and concerns, which contributed to patient advocacy. ONNs reported that they advocated for their patients by acting as a communication line between the physician and the patients. Several times they became the voice for the patients to get them where they needed to be in terms of other hospital departments. Additionally, two patient participants reported that the ONNs also referred them to community services.

Additionally, cancer patients were provided with many educational resources during their consults and some of the patients described these written resources as "a big cache of information." Weinman (1990) found that $75 \%$ of patients wanted written information about their illness rather than information given orally because the latter was not properly understood or was easily forgotten. However, findings from the present study contradicted Weinman's results. Patient participants praised the ONN for effectively explaining the information within booklets and brochures to them. The brochures were useful in laying out the information, but patients felt there was a decrease in their level of anxiety and uncertainty when an ONN explained the information to them; the explanation increased their comprehension. Providing faceto-face information to patients enhanced the personal interactions between the ONN and the patients. Cancer care has changed significantly over the past decades. Currently, there are diverse diagnostic tests, assessment programs, new medical terms, and access to numerous cancer care services. Cancer patients are bombarded with numerous pieces of information and options. Written information is one method of disseminating information to patients, but may not be as effective as face-to-face discussion between an ONN and patients. Furthermore, patients in the current study stated that having the ONN as an educational support meant they were better able to comprehend certain medical-related information. Given health literacy was a key barrier for patients, the ONNs were most effective in removing this barrier by supplementing the written materials with one-to-one interaction with the ONNs.

Findings from this current study showed that the ONNs not only provided symptom management education, but they also assessed and monitored the symptoms of their patients. Firstly, ONN provided timely and tailored symptom management education to patients. Patient participants explained that their ONNs provided various resources on symptom management and self-care during the first consult. This included information on coping with the residual and relapse warning signs, managing side effects related to the diagnostic tests, medication compliance, and daily self-care routines. Moreover, educating the patients on the importance of being aware of specific symptoms and managing them would help to decrease their anxiety and reduce wait times for physician consults. Thus, patients did not make unnecessary appointments with their physicians for every symptom they experienced because they were informed on how to manage them at home. The latter statement indicated that patients took responsibility for their health and made use of the information given to them by the ONNs. Although the ONNs facilitated patients' empowerment, it was done in a limited manner. According to World Health Organization (2009), empowerment is defined as a process in which patients understand their role, are given the knowledge and skills by their healthcare provider to perform a task in an environment that recognizes community and cultural differences and encourages patient participation.

Along with providing self-management education, the ONNs also assessed and monitored their patients' symptoms. 
By conducting a symptom assessment, the ONNs were better able to identify any major symptoms patients were experiencing that required immediate attention or changes in the treatment plan. According to Yarbro, Frogge, and Goodman (2004), poorly managed symptoms can affect cancer treatment, daily functioning, and quality of life of persons with cancer. For example, by monitoring a persistent chronic cough of the patient, the ONN can inform the physician if the cough becomes worse, which may lead to additional computed tomography (CT) scans to detect any acute or chronic changes in the lung parenchyma (Medic 8, n.d.). Thus, it was imperative for ONNs to help their patients deal with symptoms management that can adversely affect both the quality and longevity of their lives.

The ONNs also emphasized that performing screening, assessment, and evaluation of patients' condition and progress was beneficial and led to access of supportive care services for cancer patients and their families. For example, the ONNs reported that they provided access to community services such as homecare and other community agencies (i.e., churches or ethnic related agencies) to meet the physical, psychosocial, emotional, and cultural needs of the patients. By providing access to such community services, the ONNs were able to empower the patients and their families to take control of their disease and become involved in their community. They reported that providing supportive care made the diagnostic phase easier for the patients, as they had more opportunities to plan their care, organize their time, and take control of their lives and the disease. Furthermore, they explained that the patients felt supported by others from the community. Knowing that there were other patients who were experiencing similar situations or undergoing the same type of treatment for similar cancers allowed patients to reach out to one another for support and comfort. Based on these findings, it can be extrapolated that patient informational and emotional needs were met by the ONNs. Initially, patients might not have recognized community resources as a priority due to high-anxiety levels they experienced during the diagnostic assessment phase (Drageset, 2012; Moseholm et al., 2016).

\section{Limitations}

The current study acknowledged some limitations. While the sample size was small, it enabled the PI to conduct in-depth interviews, especially with the ONNs, which generated new findings about the role of the ONNs. We also used two different methods of data collection: individual interviews for patients and focus group interviews for ONNs. Triangulating the data provided more insight into the topic and strengthened the validity of the study (Burns \& Grove, 2009). With a larger sample, using multiple methods of data collection (individual interviews and focus groups) for both patients and the ONNs would have resulted in a more comprehensive analysis. Nonetheless, the findings from the study provided information about patients' empowerment and elucidated ONNs' roles.
Another limitation was that only English-speaking participants were included in this study. Excluding those patients who were not fluent in spoken English limited the examination of linguistic and cultural perspectives of patient-nurse navigator relationships. However, ONNs eliminated the health literacy barrier by providing patients with health information and included them in the decision-making process to empower them. Although participants' cultural backgrounds were not captured in this study, future research should explore language and cultural barriers of participants from diverse backgrounds and cultures.

\section{Implications}

A broad range of guidelines and practice standards currently exist for nurses and provide expert direction for best practices. With respect to oncology nurses within a Canadian setting, the results from the current study supported and illustrated how patient empowerment was enhanced in a community-based cancer centre. The domains of practice for the oncology nurse, as outlined by the Canadian Association of Nurses in Oncology (CANO) Standards of Care, Roles in Oncology Nursing and Roles Competencies document (2006), validate the core clinical functions of the ONN role identified in the current study. According to Jeyathevan, Lemonde, and Cooper Brathwaite (2017), knowledge of core competencies/ domains of practice and the extensive nurse navigation framework may be useful for decision makers, managers and healthcare providers to develop similar roles for nurses who care for patients with diseases such as mental illnesses and cardiovascular diseases. The Bi-Dimensional framework described in this study could be used to guide the content of the nursing training modules to provide a consistent and coherent patient-centred care and systematic training reflecting the clinical functions/core areas of practice of patient empowerment (Fillion et al., 2012). Lastly, patients should be empowered to take a more active role in their care and decision-making during the diagnostic phase of cancer even though it is a stressful and anxious period for them.

\section{CONCLUSION}

The complexity of cancer patients' needs requires healthcare professionals who act as navigators to direct the patients and their families through the complex healthcare system and assist them overcome barriers towards health system-level factors. Nurse navigators' roles are continuously developing and will depend on regular collaboration and communication among healthcare professionals. This study demonstrated that ONNs were able to adapt their practice to meet the emotional and supportive care needs of patient and family to empower them. Thus, collaboration among healthcare providers should be explored to examine a comprehensive outlook of interdisciplinary team functioning and how it affects standard of care for patients and their families through the cancer care continuum. 


\section{REFERENCES}

Bultz, B.D., \& Carlson, L.E. (2005). Emotional distress: The Sixth Vital Sign in Cancer Care. Journal of Clinical Oncology, 23, 6440-6441.

Cancer Care Ontario. (2010). Patient navigation pilot project. Toronto, ON: Cancer Care Ontario.

Canadian Association of Nurses in Oncology. (2006). Practice standards and competencies for the specialized oncology nurse. Toronto, ON: CANO/ACIO.

Cantril, C., \& Haylock, P.J. (2013). Patient navigation in the oncology care setting. Seminars in oncology nursing, 29(2), 76-90). WB Saunders.

Cook, S., Fillion, L., Fitch, M., Veillette, A.M., Matheson, T., Aubin, M., \& Rainville, F. (2013). Core areas of practice and associated competencies for nurses working as professional cancer navigators. Canadian Oncology Nursing Journal, 23(1), 44-52. doi:10.5737/1181912x2314452

Creswell, J.W., Plano Clark, V.L., Gutmann, M.L., \& Hanson, W.E. (2003). Advanced mixed methods research designs. Handbook of Miixed Methods in Social and Behavioral Research, 209-240.

Drageset, S. (2012). Psychological distress, coping and social support in the diagnostic and preoperative phase of breast cancer. PhD Dissertation, University of Bergen, Norway.

European Network on Patient Empowerment (n.d.). Patient empowerment_Living with chronic disease. Retrieved from http:// www.enope.eu/media/14615/a_series_of_short_discussion_ topics_on_different.pdf

Fillion, L., Cook, S., Veillette, A.M., De Serres, M., Aubin, M., Rainville, F., Fitch, M.I., \& Doll, R. (2012). Professional navigation: A comparative study of two Canadian models. Canadian Oncology Nursing Journal/Revue canadienne de soins infirmiers en oncologie, 22(4), 257-266.

Fillion, L., Cook, S., Veillette, A.M., Aubin, M., de Serres, M., Rainville, F., Fitch, M.I., \& Doll, R. (2012). Professional navigation framework: Elaboration and validation in a Canadian context. Oncology Nursing Forum, 39(1), 58-69.

Graneheim, U.H., \& Lundman, B. (2004). Qualitative content analysis in nursing research: Concepts, procedures and measures to achieve trustworthiness. Nurse Education Today, 24(2), 105-112.

Jeyathevan, G., Lemonde, M., \& Cooper Brathwaite, A. (2017). The role of oncology nurse navigators in facilitating continuity of care within the diagnostic phase for adult patients with lung cancer. Canadian Oncology Nursing Journal, 27(1), 74-80.
Medic 8. (n.d.). CT Scan. Retrieved from http://www.medic8.com/ healthguide/articles/ctscan.html

McCorkle, R., Ercolano, E., Lazenby, M., Schulman-Green, D., Schilling, L.S., Lorig, K., \& Wagner, E.H. (2011). Self-management: Enabling and empowering patients living with cancer as a chronic illness. CA: A Cancer Journal for Clinicians, 61(1), 50-62.

Moseholm, E., Rydahl-Hansen, S., Overgaard, D., Wengel, H., Frederiksen, R., Brandt, M., \& Lindhardt, B. (2016). Health-related quality of life, anxiety and depression in the diagnostic phase of suspected cancer and the influence of diagnosis. Health Quality Life Outcomes, 14(80). doi:10.1186/s12955-016-0484-9

Patton, M.Q. (2015). Qualitative research \& evaluation methods: Integrating theory and practice. SAGE Publications Inc.

Pedersen, A., \& Hack, T.F. (2010). Pilots of oncology health care: A concept analysis of the patient navigator role. Oncology Nursing Forum, 37(1), 55-60. doi:10.1188/10.ONF.55-60

Pedersen, A.E., \& Hack, T.F. (2011). The British Columbia Patient Navigation Model: A critical analysis. In Oncology Nursing Forum, $38(2)$.

Rosenbaum, E.H., \& Spiegel, D. (2002). Patient empowerment through supportive care. Cancer Supportive Care Programmes. Retrieved from http://www.cancersupportivecare.com/empower.html

Sandelowski, M. (2000). Focus on research methods. What happened to qualitative description? Research in Nursing \& Health, 23, 334-240.

Toma, J.D. (2011). Approaching rigor in applied qualitative research. The SAGE Handbook for Research in Education: Pursuing Ideas as the Keystone of Exemplary Inquiry, 405-423.

Weinman, J. (1990). Providing written information for patients: psychological considerations. Journal of the Royal Society of Medicine, 83(5), 303-305.

Wilcox, B., \& Bruce, S.D. (2010). Patient navigation: A “win-win” for all involved [Abstract]. Oncology Nursing Forum, 37(1), 21-25.

World Health Organization. (2009). WHO guidelines on hand hygiene in health care: First global patient safety challenge. Clean care is safer care. 2, Patient empowerment and health care. World Health Organization.

Yarbro, C.H., Frogge, M.H., \& Goodman, M. (2004). Cancer symptom management (3rd ed.). Sudbury, MA: Jones and Bartlett Publishers.

Zabora, J., BrintzenhofeSzoc, K., Curbow, B., Hooker, C., \& Piantadosi, S. (2001). The prevalence of psychological distress by cancer site. Psycho-Oncology, 10(1), 19-28. 